\title{
Using FIAT currencies to arbitrage on cryptocurrency exchanges
}

\author{
Tomasz Czapliński \\ Poznan University of Economics and Business, \\ Poznań, Poland \\ contact@tcraplinski.pl \\ Elena Nazmutdinova \\ Institute of Service and Industry Management \\ Industrial University of Tyumen \\ Tyumen, Russian Federation \\ nazmelena@gmail.com
}

\begin{abstract}
This paper fits in the trend of discussing the efficiency of cryptocurrency markets. Since 2008, when Bitcoin appeared on the market, arbitrageurs from all over the world have been trying to find the gaps in the markets, which will let them earn risk-free money using financial operations. Although a lot of researchers are trying to figure out arbitrage opportunities, looking at different exchanges and using different cryptocurrencies, so far hardly anyone has looked at arbitrage opportunities with the use of FIAT currencies within the same or different exchanges. This paper examines such opportunities for three different exchanges, i.e. Kraken, Bitfinex and Bitstamp - exchanges that enable trading in USD and EUR against Bitcoin at the same time. The main empirical results suggest that there are significant arbitrage opportunities on these markets. In the paper, we also show the main constraints in FIAT currencies arbitrage on cryptocurrency exchanges.
\end{abstract}

Keywords: cryptocurrency, exchange, arbitrage.

JEL Classification: D02, O17, P31

\section{INTRODUCTION}

Since 2009, when Satoshi Nakamoto (2008) published a paper about a new technology called blockchain, the whole world has been talking about it all the time, as well as about its main application, i.e. cryptocurrencies. As of this moment, Bitcoin (BTC) is the most popular cryptocurrency in the market. BTC's value accounts for more than $53 \%$ of the cryptocurrency market. It is the most recognizable, the most valuable and the most liquid currency in the world. The development of the blockchain technology 
and the appearance of new currencies have led to the establishment of cryptocurrency exchanges. At present, there are over 200 of them and their number is still growing. The existence of so many exchanges may reflect the increasing maturity of the market and, additionally, makes it possible to undertake an indepth analysis of market processes concerning cryptocurrencies.

What is one of the characteristic features of capital markets is their efficiency. The hypothesis of capital market efficiency says that prices of any goods (stocks) should be determined by currently available information and should reflect their real value in the best possible way (Fama, 1970). In accordance with the efficient market hypothesis, investors as well as economic agents behave rationally and have enough access to market information to make decisions that will maximize their profit. According to this theory, theoretically, there should be no space for investors to use arbitrage.

Among the available body of scientific literature there are a number of papers that address this issue. The first group includes articles discussing the possibility of arbitrage based on exploiting the price differences of a given cryptocurrency traded on many exchange markets or empirical studies which deal with calculations concerning the use of different cryptocurrency pairs within the same exchange (among others, Iwamura, Kitamura \& Matsumoto, 2014 or Makarov \& Shoar, 2018)

Another group of papers includes those concerning internal arbitrage for the choice of a cryptocurrency in which a given investor should take special interest in terms of supporting it (Hayes, 2014) or those analyzing arbitrage opportunities with the use of cryptocurrency-based derivatives (Hattori \& Ishida, 2018). To the best of my knowledge, no one has ever undertaken a study to examine whether it is possible to use the strategy of selling and buying cryptocurrencies with the application of traditional, socalled FIAT currencies, so that arbitrage activity could bring profits without taking any risk.

The aim of this paper is to prove that by exploiting a single virtual currency, taking advantage of the variability of traditional currencies' exchange rates, within one cryptocurrency exchange market, we can make a profit without bearing any risk. The results of this study may be used, for example, for building cryptocurrency arbitrage or trading strategies, especially for building automatic investment algorithms.

This work is in line with the existing trend in the research into capital market efficiency, the cryptocurrency market in particular. My analysis, based on the available data from three cryptocurrency exchange platforms, shows that - depending on the exchange we study - by using one hour data, arbitrage can be applied at any available examined hour of the rate of a cryptocurrency and traditional currencies. We excluded those transactions for which the rate of return is lower than their cost. Thus, the number of observations that meet such assumptions, i.e. the moments in which the strategy works and is profitable, accounts for almost 705 of the total number of observations for the given exchange market.

In our paper, we give a brief overview of scientific research into arbitrage in capital markets. We pay special attention to scientific works addressing the issue of the cryptocurrency market efficiency and arbitrage opportunities in this market. We intentionally ignored papers which concern investing in cryptocurrencies as this does not influence the research part of my study whatsoever. In the second part, we discuss assumptions based on the collected data and the possible ways of adjusting them so that they could be used as the basis for conducting research. The data come from three exchange platforms, which enable investors to trade using the BTCEUR and BTCUSD pairs. The next chapter includes the results of the study and a commentary concerning the main constraints in the practical implementation of them. The last part is a summary and conclusion, with the indication of further research areas in the field of cryptocurrency arbitrage based on traditional currencies. 


\section{LITERATURE REVIEW}

Scientific works addressing the cryptocurrency market are still rare as compared to the number and quality of papers on "traditional" economics. Nevertheless, when performing a comprehensive analysis of the existing body of literature, we may show a few main research directions. The first of them concerns the position of cryptocurrencies in the capital market. Bianchi (2017), Yermack (2013), Chiu, Koeppl (2017), Sieron (2013) and Cong, Li, Wang (2018) wonder whether cryptocurrency is money and whether it can be a payment method. Catalani, Gans (2018) and Sehra, Smith, Gomes (2017) discuss the economics of the socalled ICO (Initial Coin Offerings), i.e. the equivalent of the initial public offerings of companies. In relation to this area, a number of researchers, such as Athey (2016) or Pagnotta and Buraschi (2018), focused on the models of evaluating cryptocurrencies and cryptocurrency-based derivatives. Other authors, such as Urquhart (2016), dealt with the issue of the cryptocurrency market efficiency, especially the international efficiency of Bitcoin prices. What is quite interesting is the fact that independent camps of researchers presenting quite the opposite approaches have been formed in the field of research on the efficiency of the market under study. Scientists belonging to the first of these groups are of the opinion that the cryptocurrency market meets the tenets of the efficient market hypothesis - Brauneis and Mestel (2018), Nadarajah and Chu (2017), Vlasov and Demin (2017), Tiwari (2018), Bariviera (2017), Sensoy (2018). However, researchers from the other group, most of whom have conducted their studies relatively recently, express the opposite view - for example, Cheah (2018) or Yonghong (2018).

As regards arbitrage in the market of blockchain-based currencies, there are only a few scientific articles addressing this issue. They focus, among other things, on the possibility of arbitrage exploiting the price differences of a given cryptocurrency quoted on a lot of exchanges - Iwamura, Kitamura and Matsumoto (2014) emphasize Bitcoin's dominance in the market and the resulting limitations on arbitrage; Makarov and Shoar (2018) show that there are a number of opportunities for arbitrage exploiting price imbalances across different cryptocurrency markets; they also prove that such differences exist for a long time.

What is another research field is the use of arbitrage for selecting a cryptocurrency in which a given investor will be the most interested in the context of supporting it and, at the same time, maximizing profits from the resources they own. Particular attention should be paid to the study by Hayes (2014), who shows the possibility of establishing the most optimal currency for supporting at a given time.

The last of the research areas refers to the examination of arbitrage opportunities owing to the use of cryptocurrency-based derivatives. Hattori and Ishida (2018) point out that, in the period under study, since the creation of the first Bitcoin-based derivatives, there has been no possibility of arbitrage between these instruments.

No article was found which would enable us to state explicitly whether it is possible to use arbitrage based on pairs combined from a cryptocurrency and a traditional currency , („FIAT currency” or „FIAT money), thus, make a profit without bearing, theoretically, any exchange rate risk.

In this paper, we undertake to find the answer to the above question.

\section{METHODOLOGICAL APPROACH}

The main purpose of arbitrage is to maximize investors' profits, based on their assets, with zero risk. The above may be accomplished owing to performing transactions in two or more different markets (Grodecki, 2013). Investors exploit arbitrage when they notice that the price of an asset in a given market is different from the price of almost an identical asset in another market. If the price in the former market is higher, they sell as many of their assets as possible at an inflated price, at the same time buying in the latter market the largest possible amount of an almost identical financial instrument at a significantly lower price. In theory, the above strategy lets us conclude that it is possible to make profits with no risk attached, and 
the profit could only be potentially reduced if the transaction costs (of selling and purchasing instruments) were incurred (if issues and factors such as risk aversion, satisfaction with an economic transaction of morality are excluded from the model- see e.g. Litau, 2018). One example of instruments that could become the subject of transaction could be twin shares, quoted in two different stock markets in the same currency (Grodecki, 2013). Cryptocurrencies appear to be another very good example here. Regardless of the stock exchange they are traded in, we always deal with the same asset, which can be freely exchanged, withdrawn from one market and deposited in another one. This characteristic feature of cryptocurrencies became the basis for seeking arbitrage opportunities in different markets by the abovementioned researchers.

According to Grodecki (2015), investors distinguish two types of arbitrage in financial markets:

- Intra-market, i.e. arbitrage exploiting similar instruments within the same market, and

- Inter-market, i.e. arbitrage exploiting identical instruments in different markets or taking advantage of price differences between the base asset market and the derivative instrument market.

This paper focuses on intra-market arbitrage opportunities in three selected cryptocurrency exchanges.

In order to select appropriate exchanges which would be a source of information needed to conduct the study, we carried out an analysis of the biggest platforms in the world in terms of the value of their assets and trade volume.

The analysis, conducted on the basis of data from CoinMarketCap.com. website, showed that the biggest exchanges to be examined were: Bitstamp, Kraken, Bittrex, BTCC, Coinbase, Bitfinex, OKCoin, Bithumb, Poloniex, Gemini, Quine, Huobi, Binance, Zaif and bitFlyer. Out of these exchange platforms, I chose those for which the aggregated hourly data concerning the quotations of various cryptocurrencies are available and those in which it is possible to perform transactions in the same cryptocurrency using different traditional currencies.

The analysis of data revealed that only three exchanges fulfilled the above criteria - Kraken, Bistamp and Bitfinex. All of them offer one cryptocurrency, i.e. Bitcoin (BTC), which can be traded using two or more currencies at the same time. This was probably due to a few factors. First of all, Bitcoin is the basic currency used in the world of cryptocurrencies - according to Coinmarketcap, the market capitalization of Bitcoin accounts for over $53 \%$ of the value of all available and quoted cryptocurrencies in the world. What is more, Bitcoin is the most frequently traded currency. In all of the analyzed exchange markets, the volume of Bitcoin trade represented from $30 \%$ to $70 \%$ of the total daily trade at the platform. Thus, this is the perfect situation for conducting research on arbitrage opportunities because we prevent any criticism concerning the lack of liquidity or the impossibility to make a transaction because of the lack of knowledge of a given currency or because it is not quoted in the market.

As mentioned earlier, what is the characteristic feature of all the three exchanges is the fact that they make it possible to trade in the BTCEUR and BTCUSD pairs, irrespective of the place the user comes from or of the place from which the investor connects with the platform. The other exchanges either made only one of the above pairs (BTCEUR, BTCUSD) available or made the currency for transaction conditional on the place of stay or the place of residence that a given investor provided in the registration form.

As the next step, we obtained data concerning the hourly quotations of the BTCEUR and BTCUSD pairs from Cryptowatch service (www.cryptowat.ch). Additionally, in order to establish the hourly data of the EURUSD pair, we used data from DukasCopy website (www.dukascopy.com). 


\section{CONDUCTING RESEARCH AND RESULTS}

\subsection{Preparation for research}

On the basis of data collected I prepared a list consisting of:

- the value of the BTCEUR pair,

- the value of the BTCUSD pair,

- the value of the EURUSD pair,

to an accuracy of one hour. If none of the abovementioned three values occurred for a given moment, that moment was not taken into consideration in further research.

Taking into account the above limitations, we obtained the following three sets of data:

- Data for the KRAKEN exchange, covering the period from 7 February 2017, 21:00 UTC to 18 January 2019, 21:00 UTC,

- Data for the BITSTAMP exchange, covering the period from 7 February 2017, 21:00 UTC to 31 December 2018, 22:00 UTC,

- Data for the BITFINEX exchange, covering the period from 17 May 2018, 16:00 UTC to 31 December 2018, 17:00 UTC.

Moreover, during the preparation, we gathered information about the minimum transaction costs that would have to be incurred if it was possible to use arbitrage in the exchanges under study. For all the exchange platforms, the minimum fee for an investor performing transactions within the exchange was from $0.05 \%$ to $0.1 \%$. We also had to include additional $0.05 \%$ to $0.1 \%$ of the fee for the platform for every deposit and withdrawal or for every exchange of one virtual currency into another. On the basis of this analysis, we assumed that:

In order to show whether there is the possibility of arbitrage, we used the following algorithm for each moment: Assuming that we deal with an investor for whom US dollar (USD) is the main currency used for investment transactions, Bitcoin (BTC) was first of all purchased for US dollars (USD) at the BTCUSD rate. After that, the same Bitcoin (BTC) was exchanged into Euro (EUR) at the BTCEUR rate. As the last step, the amount obtained from selling Bitcoin for Euro was converted into US dollars at the EURUSD rate. Then, we compared by how much (in \%) the value invested in US dollars increased or decreased in relation to the obtained value.

In each case in which there is any difference, no matter whether it is positive or negative, it is possible to use arbitrage. In case the difference is positive, the investor uses the algorithm presented above. If the difference is negative, the whole procedure should be repeated backwards, i.e. first buying Euro, and ending with the BTCUSD conversion.

\subsection{Research results}

On the basis of my calculations, we concluded that, in the extreme case, it is possible to use arbitrage within a given exchange in almost $70 \%$ of the examined moments. The results for each exchange platform are presented below: 
Differences resulting from arbitrage conducted in the periods under study

\begin{tabular}{|l|c|c|c|}
\hline Exchange platform & Kraken & Bitstamp & Bitfinex \\
\hline Maximum deviation $(+)$ & $4.56 \%$ & $4.17 \%$ & $0.73 \%$ \\
\hline Maximum deviation $(-)$ & $-8.79 \%$ & $-3.28 \%$ & $-0.62 \%$ \\
\hline Number of observations - total & 9593 & 8491 & 4,655 \\
\hline Number of observations - difference $>+/-1 \%$ & 728 & 668 & 0 \\
\hline Number of observations - difference $>+/-0.5 \%$ & 1,832 & 1,701 & 9 \\
\hline Number of observations - difference $>+/-0.2 \%$ & 4,501 & 3,905 & 362 \\
\hline Number of observations - difference $>+/-0,1 \%$ & 6,560 & 5,939 & 1,514 \\
\hline
\end{tabular}

Source: Author's own work

The above table provides the following information:

- Maximum deviation $(+)$ - means the maximum observed difference that had a positive value,

- Maximum deviation (-) - means the maximum observed difference that had a negative value,

- Number of observations - means the number of moments for which the possibility of using arbitrage was examined,

- Number of observations - difference $>+/-x^{\%} \%$ - means the number of moments in which the arbitrage difference of the absolute value higher than $\mathrm{x} \%$ was observed.

Thus, the presented data show that, within one exchange, it was possible to make a profit of as much as $8.79 \%$ on a single transaction, using arbitrage with the BTCUSD, BTCEUR and EURUSD pairs. In the case of Kraken or Bitstamp, the number of observations for the absolute difference on a transaction amounting to more than $1 \%$ accounted for almost $8 \%$ of all observations. What is interesting is the fact that in none of the analyzed moments the difference on a transaction was " 0 ". The analysis was conducted with the use of hourly data. If there were data available in shorter time intervals, it would be probable that arbitrage could be used to a greater extent in a given time. With the present state of technological advancement, algorithms could perform analyses and transactions in intervals measured in seconds in a lot of exchange platforms simultaneously. However, possible delays resulting from the confirmation of transactions may cause that the state in which a transaction can be performed with zero risk could be nonexistent.

Yet the analysis of the above table allows us to make a clear conclusion- on the basis of the analyzed data, in theory, arbitrage could be used in the examined exchange platforms. In the table, we intentionally included values for which the absolute value was higher than $0.1 \%$.

\subsection{Possible constraints on the application of the research findings}

It must be remembered that the above results are theoretical calculations the application of which may be limited due to:

- the accuracy of data - data gathered for calculations come from external sources rather than from exchanges themselves. It may turn out that the data were inaccurate or did not reflect the real state in the past; 
- time needed to perform transactions - all transactions using blockchain-based currencies have to be confirmed by the community via one of two principles - proof-of-work or proof-of-stake; in the case of Bitcoin, it is proof-of-work; nowadays, it may take up to a few hours to accept a transaction with the use of BTC;

- transaction cost - most exchanges collect commission on the deposit or withdrawal of assets and on each transaction; at present, on the one hand, we observe a trend of reducing transaction costs in cryptocurrency exchanges; on the other hand, the fee policy may change any moment, which may significantly influence the level of potential profits;

- time needed to deposit or withdraw assets - now, assets are deposited in cryptocurrency exchanges through traditional bank channels; it often takes time to register resources coming from outside the exchange;

- relatively small transaction volumes - as the daily trade volume of Bitcoin is approximately $5 \mathrm{~m}$ USD, it is safe to say that this a relatively small market for institutional investors; while it provides individual investors with an opportunity to use arbitrage, the absolute obtainable amounts are not impressive.

There is another thing that must be emphasized. The above limitations occur only in the time we are writing this paper. It is highly probable that in the near future, exchanges will become the subject of interest on the part of a significantly larger number of investors; thus, they may attract more capital and become equipped with mechanisms that will make the above constraints negligible. We can already observe a trend of the instant exchange of one cryptocurrency into another within a single platform, which in turn enables faster transactions based on Bitcoin and other virtual currencies. The problem of time needed to pay investors will also slowly disappear. Originally, the biggest exchanges in the world were able to block assets to be withdrawn by a given investor for over a month. This was related to the need for adapting cryptocurrency exchanges' operations to new, previously non-existent, regulations, which were introduced by the day. At present, the average waiting time for assets to be paid to investors does not exceed 72 hours, and most platforms, for an additional fee, make payments up to 12 hours.

\subsection{Further research directions}

The above study opens new research areas in the field of arbitrage. It is natural that we should seek answers to the question what determines the fact that difference values deviate either to the positive or negative direction. What is a very important aspect from the point of view of entities using automatic stock exchange investment algorithms is the information on the timing of the process of shifting from plus to minus values and whether factors such as trade volume, time of day, day of the week, month or specific days in the year affect arbitrage opportunities.

Moreover, what could be another obvious development of my study is an attempt to use additional currency or cryptocurrency pairs in order to find out whether there are more, far more complex, arbitrage opportunities.

Finally, there is no doubt that it is necessary to conduct studies of a living organism, i.e. the cryptocurrency exchange, with the use of data flowing to the investment algorithm in real time. We have to check to what extent the response time of a given algorithm affects further possibilities of the use of the arbitrage strategy presented in this paper. 


\section{CONCLUSION}

In the paper, we proved that it is possible to use arbitrage exploiting the BTCEUR, BTCUSD and EURUSD pairs in three selected cryptocurrency exchanges, which make the quotations of Bitcoin available both in EUR and USD. The analysis of arbitrage shows that, in the period under study, there was not a single moment in which the arbitrage difference would be equal to 0 , which means that in every examined moment it was possible for investors to perform a profitable transaction without bearing any risk.

Regarding the hypothesis of the efficient capital market, it must be pointed out that either investors do not behave rationally and do not process available information to maximize their profit without exposing themselves to extra risks or the cryptocurrency market is not efficient in this respect. This situation in the market could also be due to the fact that virtual currency markets may not be mature enough to accommodate more advanced investment strategies, which are widely used in the capital markets of "traditional economics". In order to draw more conclusions with regard to this issue, we would have to carry out additional analyses trying to seek the determinants of such a state of affairs.

Further research with the use of data originating from real-time exchange systems should confirm my thesis that it is possible to use arbitrage exploiting the BTCEUR, BTCUSD, EURUSD pairs.

\section{Acknowledgement}

We would like to thank Rafał Kasperowicz, PhD, from the Poznań University of Economics and Business, for his expert assistance and methodological support in writing this paper.

\section{REFERENCES}

Athey, S., Parashkevov, I., Sarukkai, V., \& Xia, J. (2016). Bitcoin Pricing, Adoption, and Usage: Theory and Evidence. Bariviera, A. (2017). The Inefficiency of Bitcoin Revisited: a Dynamic Approach. Economics Letters, 161, 1-4.

Bianchi, D. (2017). Cryptocurrencies As an Asset Class? An Empirical Assessment, WBS Finance Group Research Paper.

Brauneis, A., \& Mestel, R. (2018). Price Discovery of Cryptocurrencies: Bitcoin and beyond. Economics Letters, 165, 5861.

Catalini, C., \& Gans, J. (2018). Initial Coin Offerings and the Value of Crypto Tokens.

Cheah, E., Mishra, T., Parhi, M., \& Zhang, Z. (2018). Long Memory Interdependency and Inefficiency in Bitcoin Markets. Economics Letters, 167, 18-25.

Chiu, J., \& Koeppl, T. (2017). The Economics of Cryptocurrencies - Bitcoin and Beyond.

Cong, L., Li, Y., \& Wang, N. (2018). Tokenomics: Dynamic Adoption and Valuation.

Fama, E. (1970). Efficient Capital Markets: A Review of Theory and Empirical Work, Journal of Finance, 25(2), 383-417. Grodecki, A. (2013). Motywy zawierania transakcji terminowych.

Hattori, T., \& Ishida, R. (2018). Do Investors Arbitrage in the Cryptocurrency Market? Evidence from the Bitcoin Futures Market.

Hayes, A. (2014). The Decision to Produce Altcoins: Miners' Arbitrage in Cryptocurrency Markets.

Iwamura, M., Kitamura, Y., Matsumoto, T., (2014). Is Bitcoin the Only Cryptocurrency in the Town? Economics of Cryptocurrency and Friedrich A. Hayek.

Litau, E. (2018). Entrepreneurship and economic growth: A look from the perspective of cognitive economics ACM International Conference Proceeding Series (pp. 143-147). http://doi.org/10.1145/3271972.3271978

Makarov, I., \& Shoar, A. (2018). Trading and Arbitrage in Cryptocurrency Markets.

Nadarajah, S., \& Chu, J. (2017). On the Inefficiency of Bitcoin. Economics Letters, 150, 6-9.

Nakamoto, S. (2008). Bitcoin: A Peer-to-Peer Electronic Cash System.

Pagnotta, E.S., \& Buraschi, A. (2018). An Equilibrium Valuation of Bitcoin and Decentralized Network Assets.

Sehra, A., Smith, P., \& Gomes, P. (2017). Economics of Initial Coin Offerings.

Sensoy, A. (2018). The Inefficiency of Bitcoin Revisited: A High-Frequency Analysis with Alternative Currencies. 
Sieroń, A. (2013). Czym jest Bitocin?, Ekonomia — Wroclaw Economic Review 19/4.

Tiwari, A, Jana, R., Das, D., \& Roubaud, D. (2018). Informational Efficiency of Bitcoin-An extension. Economics Letters 163, 106-109.

Vlasov, A., \& Demin, A. (2017). Visual methods of formalization of knowledge in the conditions of the synchronous technologies of system engineering. ACM International Conference Proceeding Series. http://doi.org/10.1145/3166094.3166098

Urquhart, A. (2016). The Inefficiency of Bitcoin. Economic Letters, 148, 80-82.

Yermack, D. (2013). Is Bitcoin a Real Currency? An Economic Appraisal.

Yonghong, J., Nie, H., \& Ruan, W. (2018). Time-Varying Long-Term Memory in Bitcoin Market. Finance Research Letters, 25, 280-284. 